\title{
REALIZING THE ASSOCIAHEDRON: MYSTERIES AND QUESTIONS
}

\author{
CESAR CEBALLOS AND GÜNTER M. ZIEGLER
}

\begin{abstract}
There are many open problems and some mysteries connected to the realizations of the associahedra as convex polytopes. In this note, we describe three concerning special realizations with the vertices on a sphere, the space of all possible realizations, and possible realizations of the multiassociahedron.
\end{abstract}

\section{INTRODUCTION}

Realizing the $n$-dimensional associahedron as a convex polytope is a non-trivial task: You are given the combinatorics of a polytope, and you are supposed to produce geometry, namely coordinates for a correct realization - such that the vertices correspond to the triangulations of an $(n+3)$-gon, and the facets to its diagonals, and a vertex lies on a facet if and only if the triangulation uses the diagonal.

The realization problem appeared first in Tamari's thesis from 1951 [39]. It was explicitly posed by Stasheff's 1963 paper [36], and first solved somewhat "by hand": As far as we know, the $n$-dimensional associahedron was constructed

- 1963 by Stasheff [36] as a cellular ball,

- 1960s by Milnor for the first time as a polytope (lost),

- 1978 by Huguet \& Tamari (see [18]: no proof given),

- 1984 by Haiman (unpublished, but see [15]), and finally

- 1989 by Lee (the first published realization: [23]).

Subsequently, more systematic construction methods emerged, among them

- the construction as secondary polytopes of convex $(n+3)$-gons,

- the construction from cluster complexes of the root systems $A_{n}$, and

- the construction as a (weighted) Minkowski sum of faces of a simplex,

all of them described in more detail below. In recent work [7, we have discovered that the realizations produced by these three families of constructions are disjoint, and that they can be distinguished by quite remarkable, geometric properties - and moreover, that there are many more realizations that seem natural as well, including the exponentially-sized

The first author was supported by DFG via the Research Training Group Methods for Discrete Structures and by Berlin Mathematical School. The research leading to these results has received funding from the European Research Council under the European Union's Seventh Framework Programme (FP7/2007-2013) / ERC Grant agreement no. 247029-SDModels. We are grateful to Carsten Lange and Paco Santos for many interesting discussions. 
family of Hohlweg \& Lange [16] [7, Sect. 4], and the even larger, Catalan-sized family of Santos [32] [7, Sect. 5].

There are many open problems and some mysteries connected to the realizations of the associahedra as convex polytopes. In this note, we describe three:

- There are several very natural, but fundamentally different constructions of the $n$ dimensional associahedron, which produce disjoint parameterized families of polytopes. How do these families lie in the realization space (defined below) of the $n$-dimensional associahedron? How do they relate?

- The associahedron constructed as the secondary polytope of $n+3$ equally-spaced points on a quadratic planar curve turns out to have all its vertices on an ellipsoid. This phenomenon extends to the permuto-associahedron and to the cyclohedron. Explain!

- Generalization of triangulations to multitriangulations leads to multiassociahedra and, more generally, to generalized multiassociahedra. Up to now, one can show that these combinatorial objects are vertex-decomposable spheres, but (how) can they be realized as convex polytopes?

Of course this note is written with the hope to clarify the situation and to explain some observations and pieces of progress related to the problems. However, some mystery remains, and perhaps this is also natural, in view of the sentence that starts Haiman's 1984 manuscript [15]:

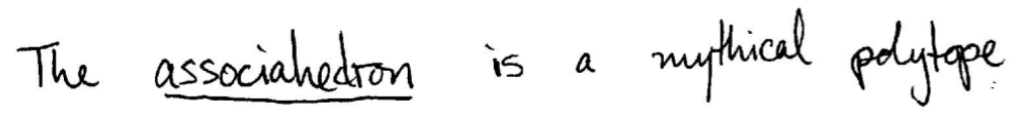

\section{Realization SPACE}

As just mentioned, there are three very natural, but fundamentally different constructions of the associahedron that may be considered to be "classical" by now:

(I) as the secondary polytope of a convex $(n+3)$-gon $Q$ by Gelfand, Kapranov \& Zelevinsky [13] [14] (see also [12, Chap. 7]),

$$
\operatorname{Ass}_{n}(Q):=\operatorname{conv}\left\{\sum_{i=1}^{n+3} \sum_{\sigma \in T: i \in \sigma} \operatorname{vol}(\sigma) f_{i}: T \text { is a triangulation of } Q\right\},
$$

where $f_{0}, \ldots, f_{n+2}$ are the vertices of an $(n+2)$-simplex,

(I) via cluster complexes of the root system $A_{n}$ as conjectured by Fomin \& Zelevinsky [10] and constructed by Chapoton, Fomin \& Zelevinsky [8],

$$
\operatorname{Ass}_{n}\left(A_{n}\right):=\left\{x \in \mathbb{R}^{n+1} \mid x_{i}-x_{j} \leq f_{i, j} \text { for } i-j \geq-1, \sum_{i} x_{i}=0\right\}
$$

for suitable $f_{i, j}>0$, and

(I) as Minkowski sums of simplices, as introduced by Postnikov in [27]

$$
\operatorname{Ass}_{n}\left(\Delta_{n}\right):=\sum_{1 \leq i<j \leq n} \alpha_{i, j} \Delta_{[i \ldots j]},
$$

for arbitrary $\alpha_{i, j}>0$, which in various different descriptions appears in earlier work by various other authors, including Shnider \& Sternberg [34, Loday [24], Rote \& Santos \& Streinu [31]. 
Some of these realizations have very striking properties, such as the vertices on a sphere (see below), or having facet normals in the root system $A_{n}$.

One would perhaps expect that "if you set the parameters right" you could get the one-and-only most beautiful realization, but a priori it is not clear, which one would that be. However, it turned out (see [7]) that these approaches yield fundamentally distinct realizations. For example, the associahedra produced as the secondary polytopes of a convex $(n+3)$-gon don't have any parallel facets, like the one in the figure,

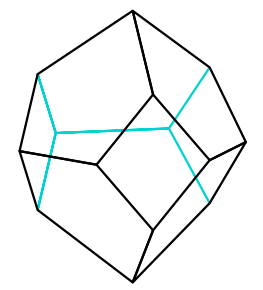

whereas the others do, typically with $n$ pairs of parallel facets that correspond to certain pairs of intersecting diagonals:
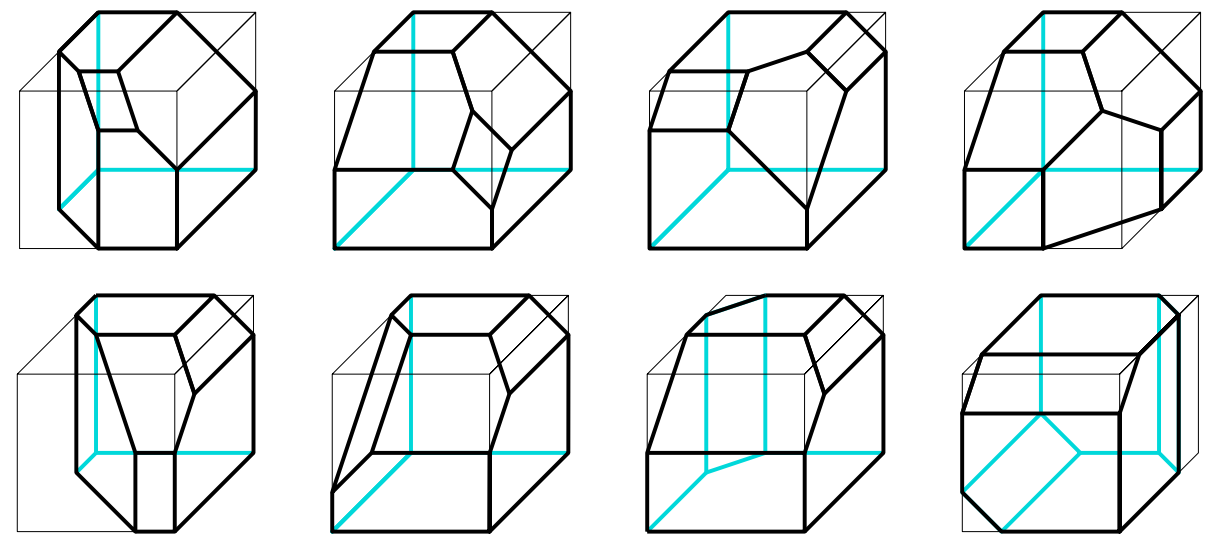

With the huge number of different realizations that are analyzed and distinguished in [7, one is led to ask a number of questions about the space of all realizations of the $n$-dimensional associahedron:

- What is the structure of the space? Is it contractible (if we divide out the action of the group of affine transformations, say)? Is it even connected?

- Do the constructions of associahedra that we know cover a large/typical part of the realization space?

o Is there any connection between the realizations? Could we get some types as a deformation/limit of other types?

The space of all realizations of (a combinatorial type of) a convex polytope is known to be a semialgebraic set defined over $\mathbb{Z}$. There are various possible definitions, which differ somewhat; if we do not identify affinely equivalent realizations and decide to only consider realizations with the origin in the interior, then the set of all such realizations - called the realization space- for an $n$-dimensional polytope $P$ with $N$ facets can be identified with 
the semialgebraic set

$$
\left\{C \in \mathbb{R}^{d \times f_{0}} \text { : the inequality system } C^{t} x \leq 1 \text { defines a realization of } P\right\} / \operatorname{Aff}\left(\mathbb{R}^{d}\right) .
$$

We refer to Richter-Gebert [29] for an extensive treatment of realization spaces of polytopes and to 30 for an introduction. The realization space of a simple $n$-dimensional polytope with $N=\frac{1}{2} n(n+3)$ facets can be seen (if we again require the origin to lie in the interior, and do not divide out a group action) as an open subset of $\mathbb{R}^{n \times N}$. It is known that realization spaces of some simple polytopes are disconnected - there are sporadic examples in dimension 4 (see [2]) and systematic constructions for high-dimensional simple polytopes [19]. But for the associahedron not much is known beyond dimension 3, where Steinitz proved in 1922 ([37]; see 29]) that the realization space of any 3-dimensional polytopes after dividing out the action of the affine group is a topological ball of dimension $f_{1}-6$.

Here is one observation that needs to be followed up: The secondary polytope construction produces a realization of the $n$-dimensional associahedron from any given convex $(n+3)$-gon. In other words, we get an associahedron from any convex configuration of $n+3$ points in the plane. The converse to this turns out to be false: "convex position" is sufficient, but not necessary for getting an associahedron.

Proposition 2.1. The secondary polytope of any configuration of $n+3$ points in the plane, which consists of all the vertices of a convex polygon and at most one point in the relative interior of any edge, is an n-dimensional associahedron.

Proof. The combinatorial structure of the triangulations of a point configuration $P$ with these properties is exactly the same as the one for a configuration $Q$ of points in convex position. If we cyclically label the vertices of $P$ and $Q$, a triangulation of $P$ corresponds to the triangulation of $Q$ consisting of the same diagonals of $P$ together with the diagonals $i, j$ such that $i, j$ is an edge of the convex hull of $P$ that has a relative interior point which does no appear on the triangulation.

Of course the point configurations of Proposition 2.1 are limit cases of strictly-convex configurations, and thus the realizations of the associahedron obtained from them are deformations of secondary polytopes of convex $(n+3)$-gons. However, the deformations do not share all their properties: Indeed, the following configuration of 6 points

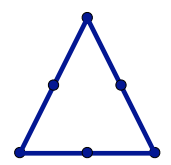

produces an associahedron that has three pairs of parallel facets - which you can never get from a hexagon [7, Thm. 3.5]. So, do we get associahedra from cluster algebras, or associahedra from weighted Minkowski sums, as limit cases of secondary polytopes? (For dimension larger than 3 we cannot expect that; cf. [7, Remark 3.6].)

And which more general, non-convex planar point configurations could still produce associahedra? 


\section{VERTICES ON A SPHERE}

The associahedron constructed as the secondary polytope of $n$ equally-spaced points on a quadratic planar curve turns out to have all its vertices on an ellipsoid, or in suitable (and still natural) coordinates even on a sphere:

Theorem 3.1. Let $p, q \in \mathbb{R}[t]$ be quadratic polynomials such that the convex curve $C=$ $\{(p(t), q(t)) \mid t \in \mathbb{R}\}$ is not a line (that is, such that $\{f, g, 1\}$ are linearly independent), and let $v_{0}, \ldots, v_{n+2}$ be equally-spaced points on $C$, that is, $v_{i}:=(f(a+i b), g(a+i b))$ for $a, b \in \mathbb{R}, a \neq 0$.

Then the secondary polytope of $Q:=\operatorname{conv}\left\{v_{0}, \ldots, v_{n+2}\right\}$, constructed according to (1) with $f_{i}:=e_{1}+\ldots+e_{i}$, has all its vertices on a sphere around the origin.

Proof. The description/construction of the secondary polytope of a convex polygon as given here is motivated by the more general setting of fiber polytopes provided by Billera \& Sturmfels [1] [40, Lect. 9]. Theorem 3.1 was observed for the special case $(p(t), q(t))=\left(t, t^{2}\right)$ and $a=1$ by Reiner \& Ziegler [28]. The more general Theorem 3.1 follows from this by simple functoriality properties of the fiber polytopes: If a polytope projection $\Delta_{n+2} \rightarrow Q$ is composed with an affine transformation of the polygon $Q$, the fiber polytope $\Sigma\left(\Delta_{n+2}, Q\right)$ changes only by a multiplication by a constant factor. An affine transformation applied to the simplex $\Delta_{n+2}$ induces the same transformation on the fiber polytope.

For the 1994 paper [28], the sphericity was discovered by chance, and established by a simple algebraic verification (with computer algebra support), establishing that the length of the GKZ vector (named after Gelfand, Kapranov and Zelevinsky)

$$
\operatorname{GKZ}(T):=\sum_{i=1}^{n+3} \sum_{\sigma \in T: i \in \sigma} \operatorname{vol}(\sigma) f_{i}
$$

does not change under flips $T \rightarrow T^{\prime}$. However, a "geometric explanation" was lacking then, and is still lacking now. The true reason is still a mystery.

This is even more deplorable as the phenomenon occurs in other instances as well. First, it does quite obviously extend to the realization of the "permuto-associahedron" that had been combinatorially described by Kapranov [22]: Indeed, the length of the GKZ vector does not change under permutation of coordinates.

Moreover calculations (Ziegler 1994, unpublished) show there is a "secondary polytope like" construction of the Bott-Taubes "cyclohedron" [4] (also known as the "type B" generalized associahedron) along quite similar lines, which again shows the same phenomenon: It produces integer coordinates for the cyclohedron, with all vertices on a sphere. This can be verified in examples using e.g. polymake by Gawrilow \& Joswig [11, it can be proved algebraically, but why is it true?

\section{Realizing the multiassociahedron}

The boundary complex of the dual associahedron is a simplicial complex whose vertices correspond to diagonals of a convex polygon, and whose faces correspond to subsets of 
non-crossing diagonals. This complex can be naturally generalized to a beautiful family of simplicial complexes with remarkable combinatorial properties. Members of this family are called simplicial multiassociahedra.

Let $k \geq 1$ and $n \geq 2 k+1$ be two positive integers. We say that a set of $k+1$ diagonals of a convex $n$-gon forms a $(k+1)$-crossing if all the diagonals in this set are pairwise crossing. A diagonal is called $k$-relevant if it is contained in some $(k+1)$-crossing, that is, if there are at least $k$ vertices of the $n$-gon on each side of the diagonal. The simplicial multiassociahedron $\Delta_{n, k}$ is the simplicial complex of $(k+1)$-crossing-free sets of $k$-relevant diagonals of a convex $n$-gon.

For example, the 2-relevant diagonals of a convex 6-gon (labeled as in the following figure, left picture) are 14,25 and 36, and the simplicial multiassociahedron $\Delta_{6,2}$ is the boundary complex of a triangle (right). The set of diagonals $\{14,25,36\}$ is not a face because they form a 3 -crossing.
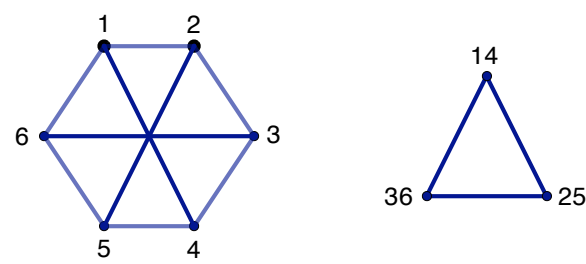

The vertices of the multiassociahedron $\Delta_{n, k}$ are given by $k$-relevant diagonals of the $n$ gon, and the facets correspond to $k$-triangulations, that is, to maximal subsets of diagonals that do not contain any $(k+1)$-crossing. For the case of $k=1$, the multiassociahedron is the simplicial complex of non-crossing sets of diagonals, which coincides with the boundary complex of the dual associahedron.

The combinatorial structure of the multiassociahedron has been studied by several authors. Apparently, it first appeared in work of Capoyleas \& Pach [5], who showed that the maximal number of diagonals in a $(k+1)$-crossing-free set is equal to $k(2 n-2 k-1)$. Nakamigawa 25] introduced the flip operation on $k$-triangulations and proved that the flip graph is connected. Dress, Koolen \& Moulton [9] obtained a reformulation of the Capoyleas-Pach result, and in particular proved that all maximal $(k+1)$-crossing-free sets of diagonals have the same number of diagonals. The results of Nakamigawa and DressKoolen-Moulton imply that the multiassociahedron $\Delta_{n, k}$ is a pure simplicial complex of dimension $k(n-2 k-1)-1$. A more recent approach for the study of $k$-triangulations, using star polygons, was given by Pilaud \& Santos [26]. In 2003, Jonsson [20] showed that the multiassociahedron is a piecewise linear sphere. Then, he found an explicit $k \times k$ determinantal formula of Catalan numbers counting the number of $k$-triangulations [21]. Additionally to the result of Jonsson about the multiassociahedron being a topological sphere, Stump [38] proved that it is a vertex-decomposable, and thus in particular shellable, simplicial sphere. See also the results by Serrano \& Stump [33].

All these results suggest that the multiassociahedron $\Delta_{n, k}$ could be realized as the boundary complex of a simplicial polytope of dimension $k(n-2 k-1)$. However, while for the classical associahedron we have many different construction methods (see above), all the 
natural approaches seem to fail for the multiassociahedron. The list of cases for which the multiassociahedron is known to be polytopal is the following. The multiassociahedron $\Delta_{n, k}$ is the boundary complex of a:

$\circ$ dual $(n-3)$-dimensional associahedron, if $k=1$;

$\circ$ point, if $n=2 k+1$;

$\circ k$-dimensional simplex, if $n=2 k+2$;

$\circ 2 k$-dimensional cyclic polytope on $2 k+3$ vertices, if $n=2 k+3[26]$;

○ 6-dimensional simplicial polytope, if $n=8$ and $k=2[3$.

Currently, the smallest open case is for $n=9$ and $k=2$. Is there a simplicial polytope of dimension 8 and $f$-vector $(18,153,732,2115,3762,4026,2376,594)$ which realizes the multiassociahedron $\Delta_{9,2}$ ?

Recently, the multiassociahedron has been generalized to a family of vertex-decomposable simplicial spheres for finite Coxeter groups, by Ceballos, Labbé \& Stump [6]. They suggest a family of simple polytopes called generalized multiassociahedra. This family includes the generalized associahedra [8] [17, and the (simple) multiassociahedra of types $A$ and $B$ (see [35] for the type $B$ description). However, no polytopal realizations of generalized multiassociahedra have been found except for the Coxeter groups of type $I_{2}(n)$, and for some particular cases in other types. The (simple) generalized multiassociahedra of type $I_{2}(n)$ are given by the duals of all even dimensional cyclic polytopes [6]. Are there polytopal realizations for generalized multiassociahedra in general?

\section{REFERENCES}

[1] L. J. Billera and B. Sturmfels, "Fiber polytopes", Annals of Math. 135 (1992) 527-549.

[2] J. Bokowski and A. Guedes de Oliveira, "Simplicial convex 4-polytopes do not have the isotopy property", Portugaliae Math. 47 (1990) 309-318.

[3] J. Bokowski and V. Pilaud, "On symmetric realizations of the simplicial complex of 3-crossing-free sets of diagonals of the octagon", in Proc. 21st Canadian Conf. Computat. Geometry (CCCG2009), 2009, 41-44.

[4] R. Bott and C. Taubes, "On the self-linking of knots", J. Mathematical Physics 35 (1994) 52475287.

[5] V. Capoyleas and J. Pach, "A Turán-type theorem on chords of a convex polygon", J. Combinatorial Theory, Ser. B 56 (1992) 9-15.

[6] C. Ceballos, J. Labbé, and C. Stump, "Subword complexes, cluster complexes, and generalized multiassociahedra", arxiv.org/abs/1108.1776.

[7] C. Ceballos, F. Santos, and G. M. Ziegler, "Many non-equivalent realizations of the associahedron", Preprint, September 2011, 28 pages; arxiv.org/abs/1109.5544.

[8] F. Chapoton, S. Fomin, and A. Zelevinsky, "Polytopal realizations of generalized associahedra", Canad. Math. Bull. 45 (2002) 537-566.

[9] A. W. M. Dress, J. H. Koolen, and V. L. Moulton, "On line arrangements in the hyperbolic plane", European J. Combinatorics 23 (2002) 549-557.

[10] S. Fomin and A. Zelevinsky, " $Y$-systems and generalized associahedra", Annals of Math. 158 (2003) 977-1018.

[11] E. Gawrilow and M. Joswig, "polymake: a framework for analyzing convex polytopes", in Polytopes - Combinatorics and Computation, G. Kalai and G. M. Ziegler, eds., Birkhäuser, 2000, 43-74.

[12] I. M. Gelfand, M. M. Kapranov, and A. V. Zelevinsky, Discriminants, Resultants, and Multidimensional Determinants, Birkhäuser, Boston, 1994. 
[13] I. M. Gel'fand, A. V. Zelevinskiŭ, and M. M. Kapranov, "Newton polytopes of principal adeterminants", Soviet Math. Doklady 40 (1990) 278-281.

[14] _ "Discriminants of polynomials in several variables and triangulations of Newton polyhedra", Leningrad Math. J. 2 (1991) 449-505.

[15] M. Haiman, "Constructing the associahedron", unpublished manuscript, MIT 1984, 11 pages; math. berkeley.edu/ mhaiman/ftp/assoc/manuscript.pdf.

[16] C. Hohlweg and C. E. M. C. Lange, "Realizations of the associahedron and cyclohedron", Discrete Comput. Geometry 37 (2007) 517-543.

[17] C. Hohlweg, C. E. M. C. Lange, and H. Thomas, "Permutahedra and generalized associahedra", Advances in Math. 226 (2011) 608-640.

[18] D. Huguet and D. Tamari, "La structure polyédrale des complexes de parenthésages", J. Combinatorics, Information 85 System Sciences 3 (1978) 69-81.

[19] B. Jaggi, P. Mani-Levitska, B. Sturmfels, and N. White, "Uniform oriented matroids without the isotopy property", Discrete Comput. Geometry 4 (1989) 97-100.

[20] J. Jonsson, "Generalized triangulations of the $n$-gon", Report from Oberwolfach Workshop "Topological and Geometric Combinatorics", April 2003, p. 11, www.mfo.de/occasion/0315/www_view

[21] _ "Generalized triangulations and diagonal-free subsets of stack polyominoes", J. Combinatorial Theory, Ser. A 112 (2005) 117-142.

[22] M. M. Kapranov, "Permuto-associahedron, Mac Lane coherence theorem and asymptotic zones for the KZ equation", J. Pure and Applied Algebra 85 (1993) 119-142.

[23] C. W. Lee, "The associahedron and triangulations of the $n$-gon", European J. Combinatorics 10 (1989) $551-560$.

[24] J. L. Loday, "Realization of the Stasheff polytope", Arch. Math. 83 (2004) 267-278.

[25] T. Nakamigawa, "A generalization of diagonal flips in a convex polygon", Theoretical Computer Science 235 (2000) 271-282.

[26] V. Pilaud and F. Santos, "Multitriangulations as complexes of star polygons", Discrete Comput. Geometry 41 (2009) 284-317.

[27] A. Postnikov, "Permutohedra, associahedra, and beyond", International Mathematics Research Notices 2009 (2009) 1026-1106.

[28] V. Reiner and G. M. Ziegler, "Coxeter-associahedra", Mathematika 41 (1994) 364-393.

[29] J. Richter-Gebert, Realization Spaces of Polytopes, Lecture Notes in Mathematics, vol. 1643, SpringerVerlag, Berlin Heidelberg, 1996.

[30] J. Richter-Gebert and G. M. Ziegler, "Realization spaces of 4-polytopes are universal", Bulletin of the American Mathematical Society 32 (1995) 403-412.

[31] G. Rote, F. Santos, and I. Streinu, "Expansive motions and the polytope of pointed pseudotriangulations", in Discrete and Computational Geometry, Algorithms and Combinatorics, vol. 25, Springer, Berlin, 2003, 699-736.

[32] F. Santos, "Catalan many associahedra", lecture at the "Kolloquium über Kombinatorik (KolKom04)", Magdeburg, November 2004.

[33] L. Serrano and C. Stump, "Maximal fillings of moon polyominoes, simplicial complexes, and Schubert polynomials", arxiv.org/abs/1009.4690.

[34] S. Shnider and S. Sternberg, Quantum groups. From coalgebras to Drinfel'd algebras: A guided tour, Graduate Texts in Math. Physics, II, International Press, Cambridge, MA, 1993.

[35] D. Soll and V. Welker, "Type-B generalized triangulations and determinantal ideals", Discrete Math. 309 (2009) 2782-2797.

[36] J. D. Stasheff, "Homotopy associativity of H-spaces", Transactions Amer. Math. Soc. 108 (1963) $275-292$.

[37] E. Steinitz, "Polyeder und Raumeinteilungen", in Encyklopädie der mathematischen Wissenschaften, Anwendungen, Dritter Band: Geometrie, III.1.2., Heft 9, Kapitel III A B 12, W. F. Meyer and H. Mohrmann, eds., B. G. Teubner, Leipzig, 1922, 1-139. 
[38] C. Stump, "A new perspective on k-triangulations", J. Combinatorial Theory, Ser. A 118 (2011) 1794-1800.

[39] D. Tamari, "Monoides préordonnés et chaînes de malcev", Doctoral Thesis, Paris 1951, 81 pages.

[40] G. M. Ziegler, Lectures on Polytopes, Graduate Texts in Mathematics, vol. 152, Springer-Verlag, New York, 1995, Revised edition, 1998; seventh updated printing 2007.

(Cesar Ceballos) Inst. Mathematics, FU Berlin, Arnimallee 2, 14195 Berlin, Germany.

E-mail address: ceballos@math.fu-berlin.de

(Günter M. Ziegler) Inst. Mathematics, FU Berlin, Arnimallee 2, 14195 Berlin, Germany.

E-mail address: ziegler@math.fu-berlin.de 